\title{
Replaceable and securely hashed keys from online signatures
}

\author{
W. K. Yip ${ }^{1 \mathrm{a})}$, A. B. J. Teoh ${ }^{1 \mathrm{~b})}$, and D. C. L. Ngo ${ }^{1 \mathrm{c})}$ \\ ${ }^{1}$ Faculty of Information Science and Technology, Multimedia \\ University, Jalan Ayer Keroh, Melaka, Malaysia \\ a) yip.wai.kuan04@mmu.edu.my \\ b) bjteoh@тти.edu.my \\ c)david.ngo@mmu.edu.my
}

Abstract: Incorporating the use of biometrics in cryptography has many advantages over the conventional use of passwords and keys. However, biometric data is non-replaceable, non-exact and requires template storage for comparison. We propose a secure method for generating biometric hash from online signature without template storage by incorporating user token and Wavelet-Fourier compression. Our results show good separation between genuine and forged signature keys.

Keywords: online signatures, biometric key

Classification: Science and engineering for electronics

\section{References}

[1] C. Vielhauer, R. Steinmetz, and A. Mayerhorf, "Biometric Hash based on Statistical Features of Online Signatures," Proc. 16th Intl. Conf. on Pattern Recognition (ICPR 2002), vol. 1, p. 10123, 2002.

[2] H. Feng and C. W. Chan, "Private Key Generation from On-line Handwritten Signatures," Information Mgmt. \& Computer Security, MCB UP Limited, pp. 159-164, 2002.

[3] C. Soutar, D. Roberge, A. Stoianov, R. Gilroy, and B. V. K. V. Kumar, "Biometric Encryption Using Image Processing," SPIE 3314, pp. 178$188,1998$.

[4] A. Juels and M. Wattenberg, "A Fuzzy Commitment Scheme," Proc. 6th ACM Conf. Computer \& Communications Security, pp. 28-36, 1999.

[5] A. Juels and M. Sudan, "A Fuzzy Vault Scheme," Proc. IEEE Int. Symp. Information Theory, ed. A. Lapidoth and E. Teletar, p. 408. 2002.

[6] W. K. Yip, A. Goh, D. Ngo, and A. B. J. Teoh, "Cryptographic Keys from Dynamic Hand-signatures with Biometric Secrecy Preservation and Replaceability," 4th IEEE on Automatic Identification Advanced Technologies, pp. 27-32, 2005.

[7] K. Liu, H. Kargupta, and J. Ryan, "Random Projection-based Multiplicative Data Perturbation for Privacy Preserving Distributed Data Mining," IEEE Trans. Knowl. Data Eng., vol. 18, no. 1, pp. 92-106, 2006.

[8] A. B. J. Teoh and D. C. L. Ngo, "Cancellable Biometrics Realization through BioPhasoring," 9th IEEE Int. Conf. on Control, Automation, Robotics and Vision (ICARCV 2006), Singapore. 
[9] SVC 2004: 1st Int. Signature Verification Competition, http://www.cs.ust.hk/svc2004/

\section{Introduction}

The traditional methods for authentication can be categorized based on "what you know" or "what you have". Password is normally used for the first category and cryptographic keys for the second. However, these two factors can be passed from one person to another, giving rise to identity frauds. Using biometric as an authentication factor not just eliminates this security risk but it is also more convenient since the biometric stays with the user all the time. For cryptographic protocols, biometric is an excellent replacement for private keys which are normally stored in tamper-proof devices. However, there are several limitations with biometric usage. In real life, biometric data do not carry high entropy as they may have high variations even within samples from the same user. Another important limitation of the biometric is that they are not replaceable. One possible way to overcome these problems is to incorporate a secret token with the biometric. This way, the user will only need to replace his token and not his biometric if the derived key is stolen. However, the biometric-token combination and key transformation must be secure and not expose secret biometric if the key is compromised.

For this paper, we use online signature as the biometric of study. The first reason for our decision is that online signatures can be easily capture from low-cost devices such as Personal Data Assistants (PDAs) and tabletbased personal computers. Secondly, it is more secure compared to static hand signatures as online signatures are more difficult to be forged.

\section{Literature Review}

Several papers on generating keys from dynamic hand signature have been reported including Vielhauer et al [1] and Feng-Chan [2] which used boundaries information for specific user to discretize real biometric input into binary keys. However, the two methods mentioned utilized parametric-based approach (using feature specific information such as the number of pen-ups, number of points, duration of signing action, velocity etc.) for biometric feature extraction. As a result, the features are limited, have varying entropies and generally short key size of around 40-50. Another problem is that the keys are not cancelable because the key is derived directly from the biometric.

Various biometric-token mixing solutions have been proposed to enable cancelable biometric key. Soutar et al. [3], Juels-Wattenberg [4], JuelsSudan [5] and Yip et al. [6] proposed to incorporate random token into the biometric to allow replaceable biometrics. There are generally two methods employed, the additive and multiplicative method. Juels-Wattenberg [4] and Juels-Sudan [5] respectively proposed fuzzy commitment (using error correction codes and XOR) and fuzzy vault which extends the former by using secret 
sharing to hide the secret. These are based on the additive method. Soutar et al [3] which used the Fourier convolution for defining the pre-designed filter is an element-wise multiplication method. Yip et al. [6] used random projection method based on user token and a novel $2 \mathrm{~N}$ discretization within fixed boundaries. Vector-wise multiplicative method using random projection is more secure as the biometric cannot be recovered using the simple inverse operations such as subtraction, de-convolution or division. However, the use of random projection introduced a loophole which enables partial recovery of the biometric feature through multiple extractions of the biometric. In particular, multiple use of the same biometrics allows an adversary to solve for the biometric vector through QR factorization as shown in Liu et al [7]. Our objectives in this paper are to design a secure method for generating replaceable biometric key using a more complex hashing (one-way) transformation without compromising on the biometric or recognition rate.

\section{Proposed Methodology}

Our proposed new method for deriving replaceable online signature hash is shown in Fig. 1. We introduce a new function-based feature extraction method (Fig. 1a), which combines the Discrete Wavelet Transform (DWT) and Discrete Fourier Transform (DFT) to obtain compact feature representation. The extracted biometric feature is mixed with a randomly generated user token $T$ using BioPhasor method [8], which "encrypts" the biometric secret in a one-way manner (Fig. $1 \mathrm{~b}$ ). A pseudo-random number generator (PRNG) is used to generate a random basis. The randomly extracted biometric feature undergoes a real-to-binary conversion using $2 \mathrm{~N}$ discretization, with the help of user-specific segment sizes that do not reveal the biometric feature. Permutation is then performed to further increase the entropy of the final key. Due to the noisy nature of biometric, error correction is then required to obtain the template key.

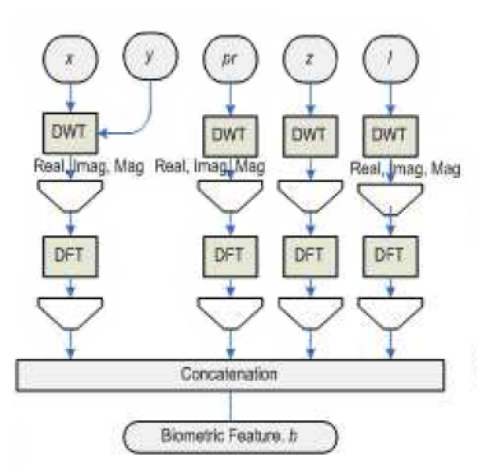

(a)

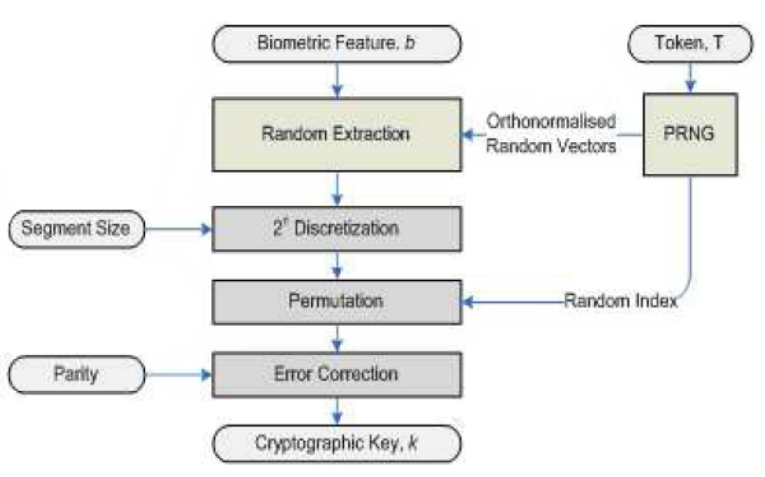

(b)

Fig. 1. Biometric feature extraction and key generation methodology

For feature extraction, we assume a pressure-sensitive pen and tablet for capturing the on-line signature signals in terms of $x$ and $y$ positional informa- 
tion, pressure information $(p r)$, pen altitude $(l)$, azimuth $(z)$, and timestamp $(t)$ for each point. Since the points are sampled consistently $(10 \mathrm{~ms})$, no resampling was performed and the pen-down segments are concatenated to form one single signal. DWT was chosen due to its good localization feature which gave a more accurate model for compression without losing important information such as sudden peaks/stops. Then DFT threshold-based compression is performed on the DWT-compressed vector to further remove high frequency coefficients, resulting in a very compact representation of the dynamic signature features. From our experiments (not shown here due to space constraint), we found that the Daubechies 6 (DB6) mother wavelet with decomposition level 2 and compression rate of $60 \%$ gave the optimal results ie. the lowest error rates between genuine and forged signature distributions. Each compressed wavelet $F$ can then be represented as DFT using the Fast Fourier Transform algorithm. Each signal is further truncated using a global thresholding method to obtain the first 18 significant amplitudes of the transforms. In our experiments, we selected the real, imaginary and magnitude components of (1) FFT (DWT $(x-y))$ (2) FFT (DWT $(p r))$ and (3) FFT $(\operatorname{DWT}(l))$ and (4) FFT $(\operatorname{DWT}(z))$ as dynamic features since they provide the lowest error rates. Finally, all the DWT-FFT compressed vectors are concatenated and normalized again to form the biometric feature, $b \in \mathrm{R}^{n}$ of length $n$.

Next, we present the online signature hashing method as follows:

1) At enrollment, generate secret random token $T$ using a PRNG and store $T$ in a tamper-proof card.

2) To compute the random basis, generate $m<n$ number of ortho-normalized random vectors $t_{i} \in \mathrm{R}_{R}^{n}$ with subscript $R$ denoting that the number is generated randomly, using $T$ as the seed.

3) Compute $h_{i}=\left[\sum_{j=1}^{n} \arctan \frac{\left(b_{j}\right)^{q}}{t_{i, j}}\right] / n$ where $q \in \mathrm{Z}^{+}$for $i=1 . . m$. Through experiment, $q=2$ gave the lowest error rate. Since $\arctan (x)+\arctan \left(x^{-1}\right)=$ $\frac{\pi}{2}$, we can rewrite $h_{i}=\left[\sum_{j=1}^{n}\left(\frac{\pi}{2}-\arctan \frac{t_{i, j}}{\left(b_{j}\right)^{q}}\right)\right] / n$, which has a more complicated transformation than random projection using random projection. In particular, the effect is a one-to-one arctan transformation of the random projection of the inverse of biometric $b$ onto bounded range of $\left(-\frac{\pi}{2}, \frac{\pi}{2}\right)$, followed by reflection of the arctan projected space along the $x$-axis and displacement of $\frac{\pi}{2}$.

4) Compute user-specific standard deviation, $s t d_{i}=$ $\sqrt{\left(\sum_{k=1}^{K}\left[h_{i, k}-\overline{h_{i, k}}\right]\right)^{2} / K}$ for $K=10$ number of training sample, and mean $\overline{h_{i, k}}$, for each element in $h$.

5) Estimate and store the number of segments in terms of bit size $n_{i}=$ $\left\{N \mid \min \left(\operatorname{abs}\left(\frac{R B-L B}{2^{N}}-\left(s t d_{i} \times 2 \times k_{\text {ind }}\right)\right)\right), N=1 . .30\right\}$ for $i=1 . . h_{\text {len }}$ where $R B=\frac{\pi}{2}, L B=-\frac{\pi}{2}$ and $k_{i n d}$, a tunable constant. Maximum value of $N$ is arbitrarily limited to 30 to avoid too many bits used for each element representation.

6) The discretized vector for random projected test input $h$ is $d_{i}=\left\lfloor\frac{\left[h_{i}-L B\right] \cdot 2^{n_{i}}}{R B-L B}\right\rfloor$ with $\lfloor$.$\rfloor being the floor function. Convert d$ to binary representation using 
Gray encoding, $k_{i}=\operatorname{gray}\left(d_{i}\right)$ to ensure that further states from the genuine region i.e. occurring with high probability from imposter test input, would have higher Hamming distances.

7) Permutate $k$ using random indices $s$ derived from $T$ via $p_{i}=d_{s_{i}}$. with $i=1 . . n^{\prime}$ where $n^{\prime}$ is the $k$ length, to provide diffusion into the key space.

8) Error correct (EC) $p$ using Reed-Solomon (RS) or binary Hamming code to obtain the template key. Majority voting is used to generate the template key. Then, a parity checksum is generated based on the template key (only this checksum is stored, not the template).

9) At verification, repeat steps 2-3 for encrypting the biometric feature and steps $6-7$ for $2 \mathrm{~N}$ discretization. Finally in step 8, append the parity checksum to the test pre-key to decode it-within the permitted threshold-into the template key $k$.

\section{Experimental Results}

We tested the proposed algorithm with Task 2 Training Database (40 users with 20 genuine and 20 skilled forgery samples per user) from [9]. For every user, the first 10 user samples are used as training database to obtain the standard deviations in the discretization step. We utilized normalized Hamming distance as the distance measurement. For random forgery (forger uses his own signature to access the system) evaluation, we compare the remaining 10 test signature keys for each user with every other user. For skilled forgery (forger simulates the genuine signature that he is impersonating), we compare the 20 skilled forgery keys with the 10 test genuine signature keys. To avoid bias in the random numbers, each experiment is repeated 5 times with different random numbers.

Table I shows the result of applying the various error correction codes on the proposed method. We indicate the Hamming EC by $\mathrm{h}(1,4)$ where one bit is corrected in every 4-bit, and the RS correction code as $\mathrm{rs}(\mathrm{m}, \mathrm{k})$ with $\mathrm{m}$ being the symbol size and $\mathrm{k}$ the code size. The Hamming distribution measurements, in terms of mean and standard deviation, are denoted as Mean-[Type] and Std-[Type] with [Type] indicating the distribution for genuine $(\mathrm{G})$, random forgery $(\mathrm{R})$ and skilled forgery $(\mathrm{S})$ hashes. The Equal Error Rates (EERs) for R and $\mathrm{S}$ forgeries before ECC are both $0 \%$. Figures 2 and 3 show the genuine and forgery hashes distribution, before and

Table I. Effect of Error Correction on BioPhasor Random Extracted and 2n Discretized Keys

\begin{tabular}{|l|l|l|l|l|l|l|l|l|}
\hline Type & $\begin{array}{l}\text { EER- } \\
\mathbf{R}\end{array}$ & $\begin{array}{l}\text { EER- } \\
\mathbf{S}\end{array}$ & $\begin{array}{l}\text { Mean- } \\
\mathbf{G}\end{array}$ & $\begin{array}{l}\text { Std- } \\
\mathbf{G}\end{array}$ & $\begin{array}{l}\text { Mean- } \\
\mathbf{R}\end{array}$ & $\begin{array}{l}\text { Std- } \\
\mathbf{R}\end{array}$ & $\begin{array}{l}\text { Mean- } \\
\text { S }\end{array}$ & $\begin{array}{l}\text { Std- } \\
\text { S }\end{array}$ \\
\hline $\begin{array}{l}\text { Without } \\
\text { Correction }\end{array}$ & 0.000 & 0.000 & 0.038 & 0.018 & 0.418 & 0.010 & 0.210 & 0.010 \\
\hline $\begin{array}{l}\text { With } \\
\text { h(1,4) }\end{array}$ & 0.000 & 0.000 & 0.009 & 0.007 & 0.421 & 0.010 & 0.110 & 0.010 \\
\hline $\begin{array}{l}\text { With } \\
\text { rs(4,9) }\end{array}$ & $\mathbf{0 . 0 0 0}$ & $\mathbf{0 . 0 0 0}$ & $\mathbf{0 . 0 0 1}$ & $\mathbf{0 . 0 0 2}$ & $\mathbf{0 . 4 2 2}$ & $\mathbf{0 . 0 0 9}$ & $\mathbf{0 . 0 7 2}$ & $\mathbf{0 . 0 1 4}$ \\
\hline
\end{tabular}




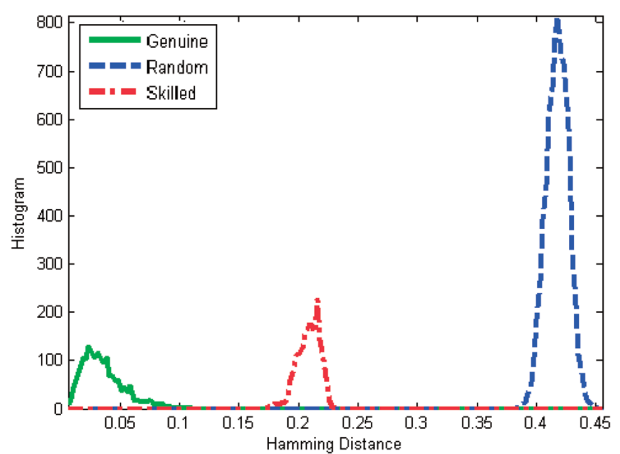

Fig. 2. Distribution before EC

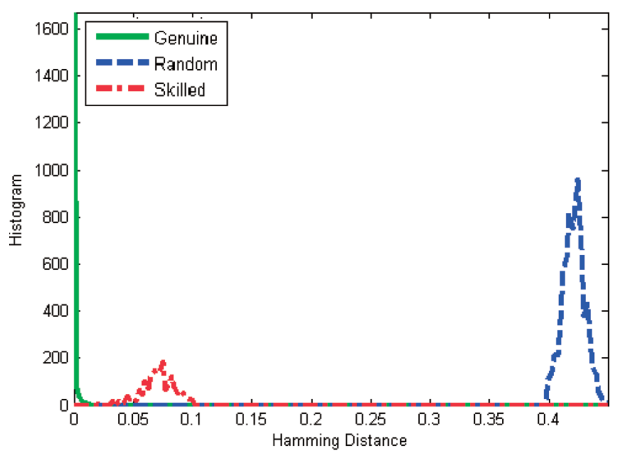

Fig. 3. Distribution after EC

after EC respectively. For both cases, we can see that there are clear separations among all the distribution. After EC, most of the genuine bit-strings are corrected to the exact template key, as indicated by the $0.1 \%$ Hamming distances obtained using using $\mathrm{rs}(4,9)$.

\section{Security Analysis}

The security strength of the proposed scheme lies in the irreversible random extraction of biometrics information via BioPhasor and a lossy real-to-binary discretization process. We proceed with sketch of the security proof.

Proposition: If the key and the genuine token $T$ are known, recovering the biometrics feature $b$ exactly requires exponential time i.e. intractable problem.

Sketch of Proof: The random vectors $t_{i}$ are known since token $T$ is known. Firstly, we attempt to recover $b$ from the interim BioPhasor vector $h$. We can form the system of equations $h_{i}=\left[\sum_{j=1}^{N} \arctan \frac{\left(b_{j}\right)^{q}}{t_{i, j}}\right] / n=\left[\sum_{j=1}^{N}\left(\frac{\pi}{2}-\right.\right.$ $\left.\left.\arctan \frac{t_{i, j}}{\left(b_{j}\right)^{q}}\right)\right] / n$ where $q \in \mathrm{Z}^{+}$and $i=1 . . m<N$ where $N$ is the length of biometric feature vector. Due to the presence of arctan operation, solving the system of equations cannot be straightforwardly performed using QR factorization. Also, since $\tan \left(h_{i}\right) \neq \sum_{j=1}^{n}\left(b_{j}\right)^{q} t_{i, j}^{-1}$, we cannot linearly transform the system of equations into Gaussian eliminated form. Since $i=1 . . m<N$, the system has infinite solutions. It is clear that solving this system requires exponential time, hence solving for the biometric $b$ from $h$ is an intractable problem. Second, we attempt to recover the $b$ from the binary key. Since the 
element space of $h$ is quantized into $2^{n_{i}}$ segments and subsequently permutated by bit, the computation complexity in recovering indices of the quantized space is equivalent to brute force eg. $2^{n_{1}+n_{2} . .+n_{m}}$. From our experiment, the length of key produced is around 2000 bits; hence recovering $b$ from our method is an intractable problem.

\section{Conclusion}

The objective of this paper is to propose an online signature hash generation method which is robust against recovery of the biometric from compromised keys. We have shown in our security analysis that recovery of the exact biometric requires exponential time. The experimental results confirmed that genuine keys are distinguishable from the forgery keys, and are exactly reproducible. 